\title{
Model undergraduate integrated optics laboratory
}

\section{Biswajit Das, Lawrence Hornak, Michael Lemmon}

Biswajit Das, Lawrence Anthony Hornak, Michael D. Lemmon, "Model undergraduate integrated optics laboratory," Proc. SPIE 2525, 1995 International Conference on Education in Optics, (13 October 1995); doi: $10.1117 / 12.224007$

SDIE Event: SPIE's 1995 International Symposium on Optical Science, Engineering, and Instrumentation, 1995, San Diego, CA, United States 


\title{
A Model Undergraduate Integrated Optics Laboratory
}

\author{
B. Das and L. A. Hornak \\ Microelectronic Systems Research Center \\ Department of Electrical and Computer Engineering \\ West Virginia University \\ Morgantown, WV 26506. \\ M. Lemmon \\ Department of Electrical Engineering \\ University of Notre Dame \\ Notre Dame, IN 46556.
}

\begin{abstract}
This paper describes recent efforts at developing a low cost undergraduate laboratory demonstrating current technologies used in integrated optics. The lab was targeted at undergraduate seniors to acquaint them with the role of integrated optics in the communications industry. This project examined two different types of integrated optical devices (1) glass waveguide devices using ion-exchange technique, and (2) polymer devices. Both device types can be implemented using equipment and materials already available in a standard university integrated circuit (IC) fabrication facility. The results of this project identify the polymer fabrication technology as best suited for the average environment found in undergraduate laboratories.
\end{abstract}

\section{Introduction}

Technological innovations in long haul fiber optic communication networks have generated a need for integrated optical circuits (IOC). Such circuits integrate optical waveguide devices such as beam splitters, polarizers, directional couplers etc. with conventional opto-electronic devices such as phase modulators, intensity modulators, switches, on the same substrate. IOCs are more compact, have lower drive voltages, lower optical losses and higher modulation frequency than discrete component systems; and as a result they are finding increasing applications in a number of communication systems. Thus, it is becoming more and more important that graduating seniors in Electrical Engineering have a working understanding of such devices. This paper documents recent efforts at the University of Notre Dame and West Virginia University to develop such a laboratory program. This project was carried out with the partial financial support of a grant from the National Science Foundation's (NSF) Leadership in Laboratory Development (ILI-LLD) program between 1992 and 1994.

The remainder of this paper is organized as follows. Section 2 discusses fundamental waveguide photonic concepts which were taught to students. Section 3 discusses our efforts at identifying waveguide fabrication technologies that matched the technical level of the average undergraduate senior. Sections 4 and 5 discusses laboratory experiments and section 6 discusses future plans for this project. 


\section{Photonics Course Development}

The lecture component of this course was targeted at undergraduate seniors. It was assumed that the students had the standard background in electro-magnetic (EM) theory up to the formulation of Maxwell's equations and a network/systems background which included the concepts of transfer functions, eigenvalues, and eigenvectors. These pre-requisites are primary components of most ABET accredited programs. Also, with faculty expertise spanning the areas of devices and systems, special effort was made to address device fabrication technologies and system level design issues in an integrated manner.

The photonics course was designed to revolve about a careful analysis of optical slab waveguides. A slab waveguide is a thin dielectric film whose index of refraction is greater than the index of refraction of the surrounding material. Such structures trap light within the thin film, thereby acting as a "guide" which conducts the light along the film. The lecture component of the course provides students with the analytical tools to characterize and predict the performance of simple waveguide structures. The course starts by reviewing Fresnel's equations describing the reflection/refraction of light at a plane dielectric interface. Fresnel's equations provide sufficient background to discuss the geometric theory for planar waveguides. The course then examines the process of wave guiding by solving Maxwell's equations. This part of the course allows students to compute elementary waveshapes for simple planar structures using MatLab and provides sufficient background to permit a quantitative discussion of coupled-modes in perturbed waveguides. With the background in planar waveguiding and mode coupling, the course is then able to discuss passive waveguide devices, active devices such as detectors, lasers/LED's, and active waveguide devices such as modulators. A listing of the topics and the amount of time spent in each is itemized below.

Topic / Time

Fresnel Equations / 1 week

Slab Waveguides / 2 weeks

Cylindrical Waveguides / 1 week

Mode Coupling / 1 week

Passive Waveguide Devices / 2 weeks

Detectors/Lasers/LED / 3 weeks

Active Waveguide Devices / 2 weeks

Optoelectronic Integrated Circuits / 1 week

The photonics course without the laboratory was first offered in the spring of 1993 . A total of 15 students took the course out of which 11 were senior undergraduate students and 4 were first year graduate students. The course was jointly taught by B. Das and M. Lemmon and was received well by the students. The textbook used in this course was "Guided Wave Photonics" by Buckman which provided a good overview of all the topics listed above. The following books were used as recommended texts for the course : "Classical Electrodynamics" by Jackson, "Fourier Optics" by Goodman and "Applied Optics" by Banjaree and Poon.

\section{Integrated Optics Laboratory Development}

There is a laboratory component associated with the lecture component of the course. The lab provides students with an opportunity to experimentally validate the concepts learned in the classroom. The laboratory 
described below was developed at the University of Notre Dame with the partial financial support of the National Science Foundation's ILI-LLD program.

\subsection{Selection of Fabrication Technology}

This section discusses fabrication methods used in making thin film slab waveguides. In order to be adopted in an undergraduate lab, the fabrication technology need to be safe, inexpensive, easy to use, and provide devices of moderate quality. Two different types of devices were investigated; glass waveguides using potassium nitrate $\left(\mathrm{KNO}_{3}\right)$ ion-exchange technique and polymer waveguide devices.

\subsubsection{Ion-exchange technique}

The ion-exchange technique creates a waveguide by diffusing potassium ions into a soda-lime glass substrate and replacing the sodium ions, thereby increasing refractive index in the diffused region. We duplicated experiments discussed in [1]-[4] to determine whether or not ion-exchange technologies were appropriate in an undergraduate lab setting.

Fig. 1 schematically shows the equipment in which the ion-exchange process was carried out. The basic steps involved are : melting potassium nitrate at $385 \mathrm{C}$, immersing the glass slides in the molten potassium nitrate, and perform ion exchange for 1 to 64 hours.

\subsubsection{Polymer process}

Polymer waveguides are fabricated by spinning a layer of optical polymer on a substrate. There are many types of polymers that can be used for this process; such as polyurethane, polystyrene, epoxy, organometallic solutions, and photoresist. The polymer used by this lab was positive photoresist typically used in VLSI fabrication. The decision to use this material was based on the availability and low cost of the material. The type of positive photoresists used were Shipley Microposit S1400-17 and S1400-27. Experiments were also performed with negative photoresist (OCG VHR-2), but were found be less desirable. The waveguide fabrication process invovles coating the substrates with hexmethyldisilizane (HMDS), spinning on the photoresist, and then baking the photpresist to harden it to form a solid waveguide, as shown in Fig. 2.

\subsection{Evaluation of Fabrication Technologies}

The ion-exchange process was found to be less suitable for the undergraduate laboratory to the polymer process for the following reasons.

First, the process was highly toxic and special care needs to be taken to prevent inhalation of $\mathrm{KNO}_{3}$ fume and skin contact with $\mathrm{KNO}_{3}$. There is also the additional risk of explosion in case the temperature of $\mathrm{KNO}_{3}$ rose above $400 \mathrm{C}$. The polymer process in contrast is much safer.

Another major factor considered was the amount of time necessary for the fabrication of the waveguides. The ion exchange technique involves diffusion of ions at relatively low temperatures which is an inherently slow process, requiring diffusion times of 24 hours or more for waveguides with reasonable depths. Polymer spinning and curing on the other hand is a relatively quick process.

Slide breakage was also a factor in selecting the fabrication process. The ion exchange process has many more chances for shattering of slides, such as temperature difference between glass slide and KNO3 melt, 
inadequate cooling rate, contact of glass slides with metallic beaker during raising and lowering, etc. On the other hand, slide breakage was relatively rare for the polymer process.

Another advantage of the polymer waveguide devices is that they are not permanent and hence can be redone in case of mistakes. The baked photoresist is fairly rugged, however, it is possible to remove the polymer with photoresist remover in case of mistakes. This allows recycling of substrates and cut down of operation costs. In contrast, the ion-exchange process is permanent.

The contrast between the waveguide and the substrate is much greater for polymer waveguides than for the glass waveguides, an useful feature for the fabrication of patterned waveguide devices and for the alignment of optical fibers.

The polymer waveguides have greater difference of refractive index than the ion exchange glass waveguides, thus enabling tighter light confinement.

The only area where the ion-exchange process was found to be superior to the polymer process was in waveguide quality. Ion-exchange waveguides generally exhibited significantly less dispersion and showed clearer $\mathrm{m}$-lines than the polymer waveguides. However, the quality of the polymer waveguides was sufficiently good for carrying out the experiments.

\subsection{Description of Laboratories Developed}

The laboratories developed under this program have two components : i) the fabrication laboratory and ii) the characterization laboratory. The fabrication laboratory was housed in the existing microelectronic fabrication laboratory and contained the equipment necessary for waveguide and device fabrication a list of which is shown in Table I. The photoresist spinner and the ion exchange system were installed inside fume hoods to ensure student safety, which was a major consideration during laboratory development. In the fabrication laboratory, used equipment were used for non critical steps to keep the development cost low. The characterization laboratory was housed in the existing optical signal processing laboratory. The laboratory sessions associated with waveguide characterization and device testing were conducted in this facility. Two student work stations were setup consisting the equipment necessary for waveguide and device characetrization, a list of whcih is shown in Table I. The total cost for the development of the fabrication and the characterization laboratory was around $\$ 50,000$.

\section{Laboratory Sessions}

The laboratory component of the course was offered for the first time in spring 1994. A total of 6 senior undergraduate students were enrolled in the course and they worked in groups of 3 in the laboratories. The following experiments were performed by the students.

\subsection{List of Experiments}

\section{Lab 1 Index of Refraction Measurement}

In this laboratory the students experimentally measured the index of refraction for a number of microscope glass sildes that were used for substrates for the follwoing experiments. The students cleaned the 
microscope glass slides and measured their refractive indices by the Brewster angle technique [6].

\section{Lab 2 Fabrication of Photoresist Slab Waveguides}

In this laboratory the students fabricated planar photoresist waveguides on the glass slides characetrized in the previous experiment. The students fabricated waveguides of three different thicknesses ranging from 1.5 microns to 5 microns which required single and double spin coatings. The students were provided with the photoresist data sheet from which they selected spin speed and baking temperatures to obtain the desired results.

\section{Lab 3 Slab Waveguide Characterization}

The waveguides fabricated in lab2 were characterized for their depths and refractive indices which allowed the characterization of the fabrication process as well as the waveguide performance in terms of dispersion and loss. The technique used for the characterization is a variation of the prism coupling method described by Ulrich and Torge [5]. This technique experimentally determines the depth and index of refraction of the waveguide, which can then be compared to analytic models to determine the qualities of the two fabrication processes.

The physical set-up for the waveguide characterization is shown in Fig. 3 and is based on the work described in [6]. The slide is taken and clamped against a metal plate and then pressed against the coupling prisms. The whole system rests on an xy-translation stage which has been mounted on a rotation stage which also has an observation screen mounted on it. The laser beam is aimed into the corner of the prism which is the coupling spot.

The incoming light is linearly polarized and a half-wave plate is used to adjust the plane of polarization so that only the TE or TM modes are excited in the waveguide. In addition to this, the laser beam was collimated and a focusing lens was used to make the coupling spot as small as possible to increase the coupling efficiency.

The setup is adjusted to keep the coupling spot stationary on the prism base while the setup is rotated over the required angular range. It is necessasry to get the coupling spot near the corner of the prism to maximize the amount of incident light coupled into the waveguide. Once this is accomplished, the angles of propagation, $\alpha_{\mathrm{m}}$, of the modes in the waveguide are determined. These mode angles are obtained by measuring the beam's incidence angle, $\theta_{\mathrm{m}}$, which is then converted to $\alpha_{\mathrm{m}}$ using simple geometry [6].

After the coupling spot has been appropriately adjusted, the whole setup is slowly rotated and the output light is projected onto the screen. At first, for small beam incident angle, the light travels through the waveguide and couple into the substrate. The substrate modes are vey easy to see as broken lines in the slide. These modes are also projected onto the screen as series of sharply defined bright lines, called $m$-lines. As the setup is further rotated, the substrate modes grow fainter and the lines become more widely spaced until they disappear altogether. If the incidence angle is further increased, light then begins to couple into the waveguide rather than the substrate. These waveguide modes appear as a "broken" streak of light across the slides which also project a set of m-lines.

Once the range of modal coupling was determined, it was necessary to observe the number of modes supported by the guide and their associated $\alpha_{\mathrm{m}}$ by scanning through all the modes several times. The mode with the largest incidence angle was labeled mode zero. Because of the nature of the modal coupling, it is often necessary to adjust the clamping pressure to observe the modes clearly which tends to broaden and shift the modes. 


\section{Lab 4 Fabrication of Integrated Optical Device}

The goal of this laboratory was to fabricate a photoresist directional coupler on a $\mathrm{SiO}_{2} / \mathrm{Si}$ substarte . This particular device was chosen because it is easier to fabricate and provide the students with insight into the coupled mode theory. The $\mathrm{SiO}_{2} / \mathrm{Si}$ substrates were chosen so that light could be coupled in and out of the device with optical fibers using V-grooves which is an important tool for alignment in optoelectronic packaging. Schematic diagrams of the sunstrate with fiber optic coupling and the directional coupler are shown in Figures $4 \mathrm{a}$ and $4 \mathrm{~b}$. The device consists of two waveguides of widths of $8 \mu$ separated by a distance of $4 \mu$, with an underlying layer of photoresist which acts as the coupler. The students were supplied with the $\mathrm{SiO}_{2} / \mathrm{Si}$ substrates with the V-grooves etched on, on which they fabricated the directional couplers. As discussed in section 5, this device was found to be too difficult for undergraduate students due to the critical alignment requirements for single mode fibers. A modified device is currently under investigation which will be discussed in section 6.

\section{Lab 5 Characterization of Integrated Optical Device}

In this laboratory the students characterized the directional coupler fabricated in the previous experiment. Light was coupled in and out using single mode optical fibers utilizing the V-grooves for alignment. The intensity of the input and output light were measured and analyzed.

\subsection{Discussion of Classroom and Laboratory Experience}

The laboratory component of the course was offered for the first time in spring 1994. A total of 6 senior undergraduate students were enrolled in the course and they worked in groups of 3 in the laboratories. The students have all the prerequisites for understanding the lecture materials and sufficient laboratory experineces to perform the lab experiments. Both the lecture and the lab components of the course went smoothly except for lab 5 as described later. In the lecture component, the students were graded on three 1-hour exams and a comprehensive final exam as well as weekly homework assignments. In the laboratory, the students performed the five experiments described earlier. The students had no difficulty in the fabrication of the devices, however, during device characterization, each student went through a learning process for proper alignment of the optics.

There were some problems encountered during the characterization of the directional couplers. None of the directional couplers fabricated by the students (each group fabricated a set of three devices) were found to be working. Close inspections of the devices revealed misalignments of the order of a micron between the V-groove and the waveguide axes. Such misalignment is fatal for single mode fibers in which the optical power is almost completely confined within a radius of a micron from the axes. While graduate students were found to be quite capable of such alignments, it was found to be over ambitious for undergraduates. While sinlge mode devices have the advantages of analytical computation, fabrication of such devices appear to be unsuitable for undergraduate courses. Based on this experience, a modified inetegarted optical device is currently being developed at West Virginia University, as described in section 5. 


\section{Modified Integrated Optical Device}

Based on our previous experience, an integrated optical device most suitable for an undergraduate laboratory require to be multimoded and simple in configuration. The modified integrated optical device selected is a polymer waveguide $\mathrm{Y}$ branch power divider. A schematic cross section of the device is shown in Fig. 5a. This particular device was selected for its ease of fabrication and its ability to teach device fabrication technology including masking and alignment, as well as the current fiber optic coupling techniques. The waveguide dimensions chosen are $30 \mu \times 30 \mu$, which allows easier alignment with as well greater coupling from the multimode fiber. This will ensure sufficient light coupling from the fiber to the waveguide to carry out the experiments even in case of some misalignment. Since such waveguides are difficult to fabricate using photoresist, we have chosen ULTRADEL 9020D (Amoco Chem) to be the polymer for implementing the waveguides. This optical polymer is commercially available and is designed for low loss $(\alpha=0.32 / \mathrm{cm}$ at 633 $\mathrm{nm}$ ). This particular polymer is also photoimageable which allows similar processing steps as for photoresist waveguides. The processing steps for the $\mathrm{Y}$ - branch power divider is shown in a flow chart in Fig. 5a.

\section{Future Course Offerings}

The integrated optics course and the modified laboratory are tentatively planned to be offered in the spring of 1996 at West Virginia University. In the lecture component of the course, we plan to introduce computer sofytware for waveguide analysis. Such a software package has been recently acquired at West Virginia Unievrsity which can calculate optical field distribution for complex waveguide structures [7]. In the laboratory component we propose to keep lab1 - lab3 the same. For lab4, the students will fabricate the Ybranch power divider on $\mathrm{SiO}_{2} / \mathrm{Si}$ substrates with $\mathrm{V}$-grooves for fiber optic coupling. In lab 5, the students will be asked to characterize the power splitter by V-groove fiber optic coupling as well as fiber end fire coupling as shown in Fig. 5b. The latter is chosen to emphasize the critical coupling requirements in phtonic systems.

\section{Discussion}

This project has successfully demonstrated the feasibility of an undergraduate laboratory in the area of integrated optics and has developed a set of laboratory experiments that can be duplicated at other institutions. The cost of laboratory development was kept around $\$ 50,000$ to enable small and midsize universities to offer such programs. After investigation of a number of different technologies it has been established by this project that the polymer waveguide device technology is the most suitable for an undergraduate laboratory program. The waveguide fabrication and characetrization processes have been well characterized and have shown to be achievable by senior undergraduate students. The integrated optical device developed in this project will provide students with hands on experience on device fabrication technology as well as expose them to the current industry standard for alignment with optical fibers. 
Table I Equipment List for the Integrated Optics Laboratory

Fabrication laboratory : photoresust spinner + fume hood, mask aligner, photoresist baking oven, inspection microscope, acid bench, solvent bench, masks, ion exchange system + fume hood.

Characterization Laboratory : optical breadboards, $1 \mathrm{~mW}$ HeNe lasers, posts, xy translation stages, tilt/rotation stages, micropositioners, fiber optic couplers, fiber positioners, halfwave plates, power meters, lens and mirrors.

\section{REFERENCES}

[1] Lagu and R. Ramaswamy, Appl. Phys. Lett. 45(2), 117-118 (1984).

[2] R. Ramaswami and S. I. Najafi, IEEE Journal of Quantum Electronics QE-22, 883-891 (1986).

[3] S. I. Najafi and M. J. Li in Photonic Networks : components and applications, World Scientific Publishing, 46-55 (1991).

[4] Pal and Kersten, IEEE Trans. on Education, E-28, 22-52 (1985).

[5] R. Ulrich and R. Torge; Applied Optics 12, 2901 (1973).

[6] W. LaMonaca; Design and Fabrication of Low Cost Polymer Directional Couplers, MS thesis, Univ. of Notre Dame (1994).

[7] BPM-CAD by Optiwave Corporation, Canada. 


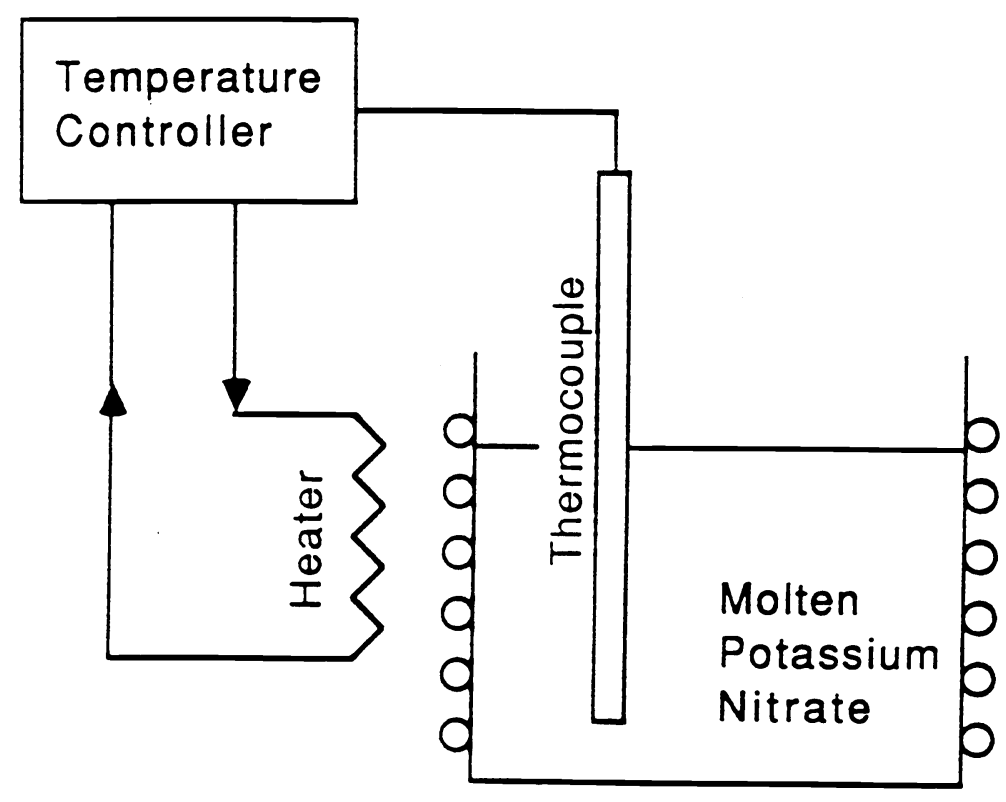

Fig. 1 Schematic diagram of the ion-exchange apparatus.

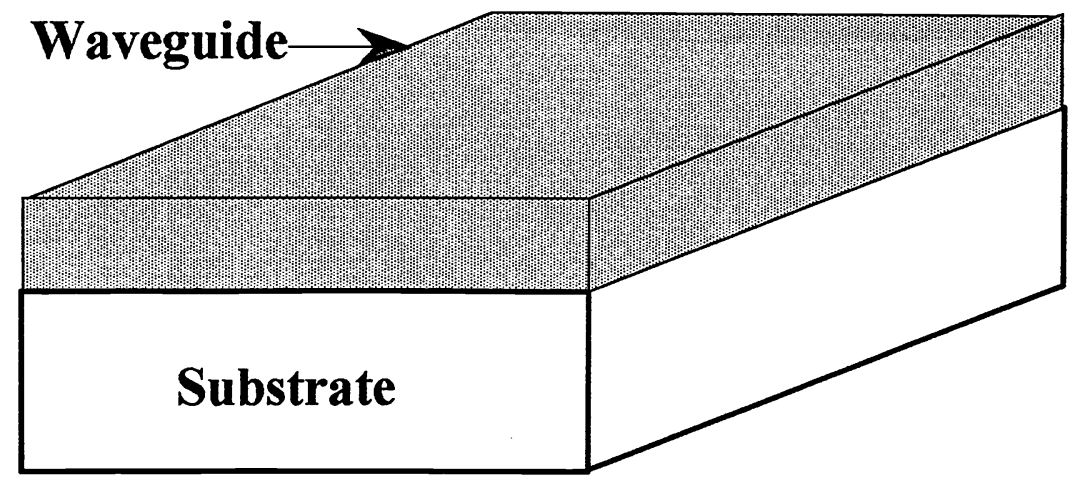

Fig. 2 Optical waveguide formed by spinning photoresist on glass substrate. 

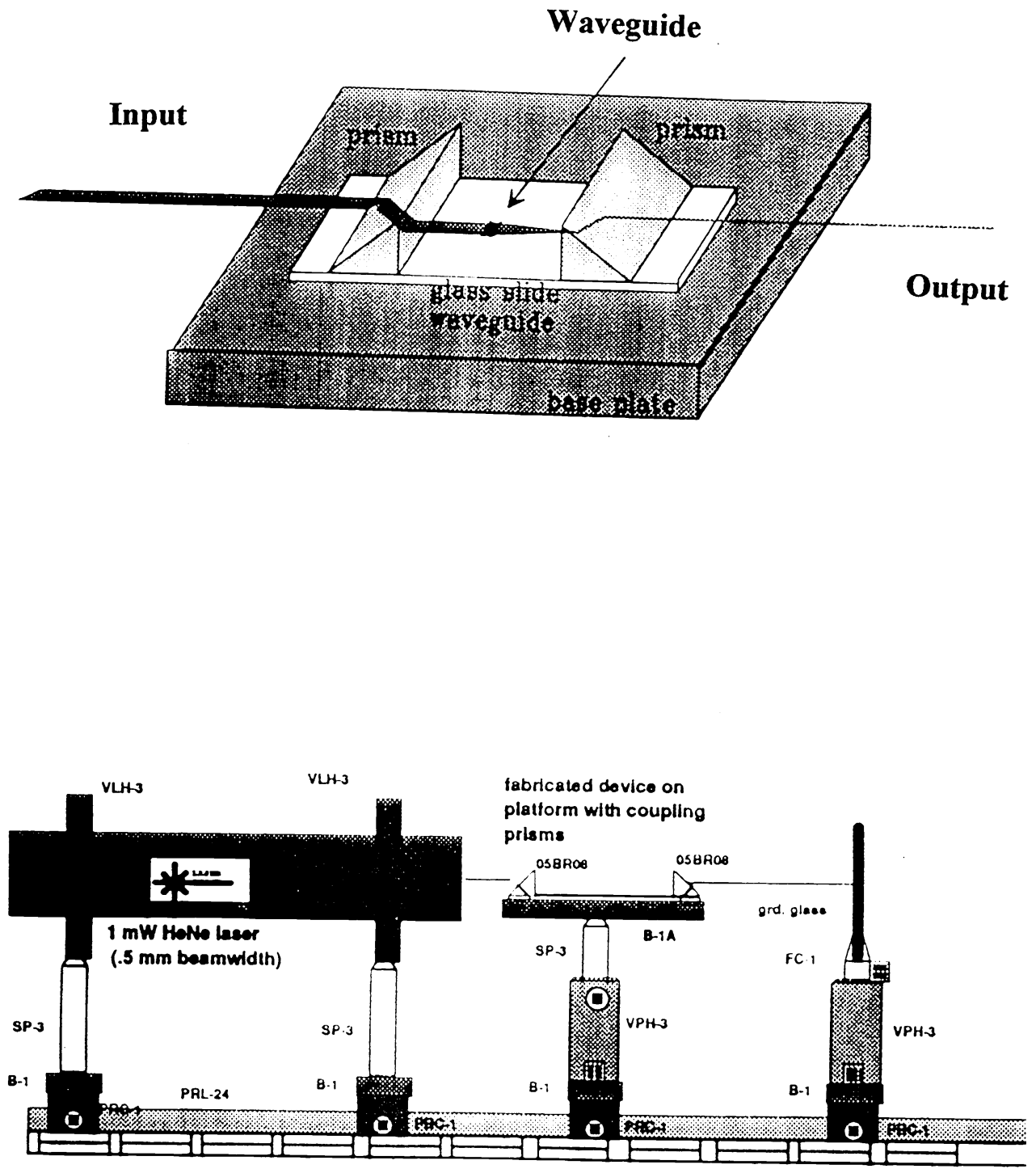

Fig. 3 Experimental setup for waveguide characterization. 

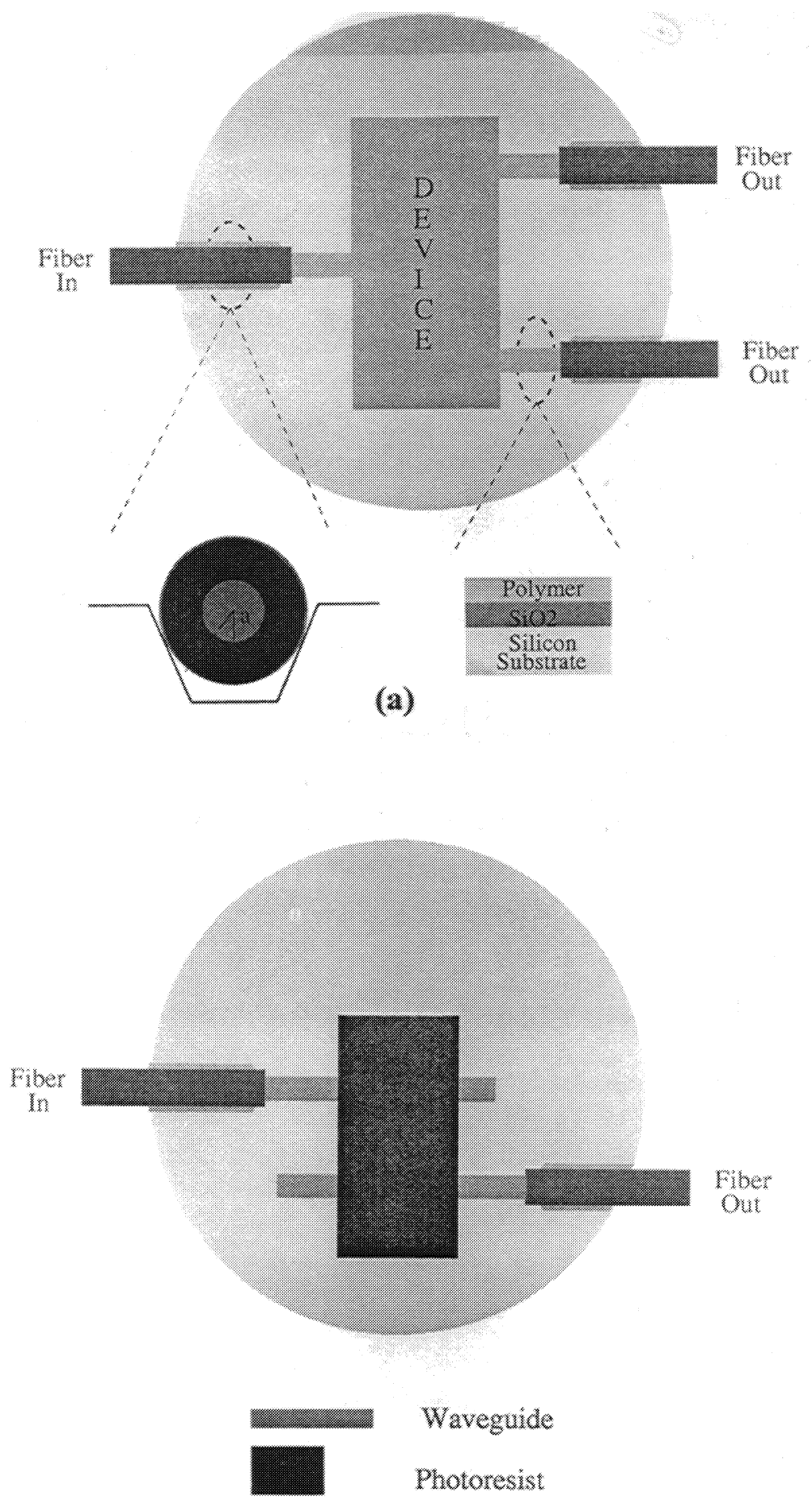

(b)

Fig. 4 Schematic diagram of (a) substrate with V-grooves for fiber optic coupling and (b) photoresist directional coupler. 


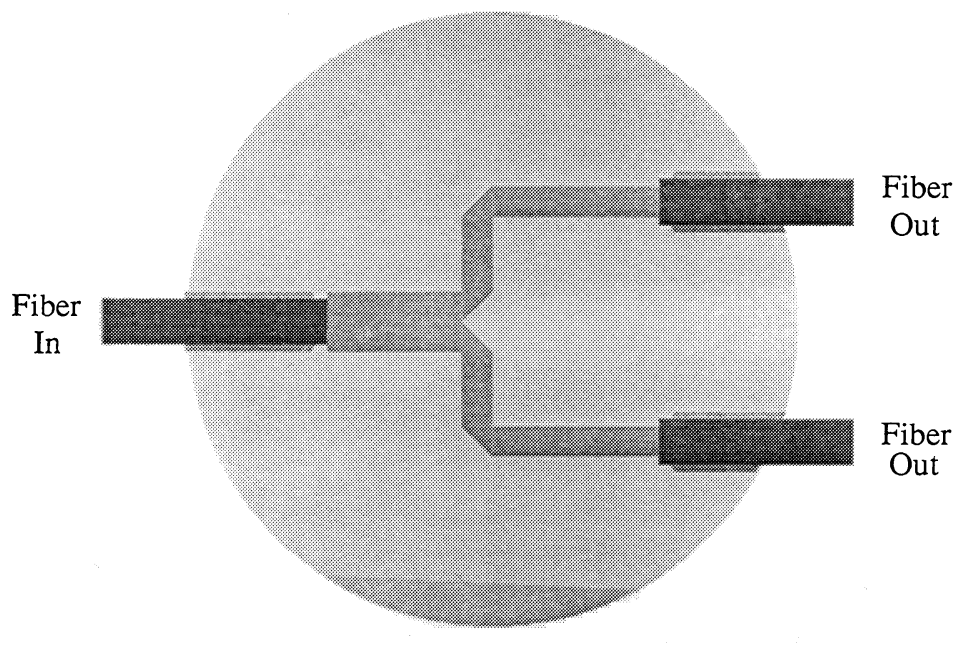

\section{Processing Steps $(30 \mu \mathrm{m})$}

1. Dehydrate substrate at $200^{\circ} \mathrm{C}$ for 30 minutes

2. Apply polymer. Spin at $500 \mathrm{rpm}$ for $30 \mathrm{sec}$ followed by $1000 \mathrm{rpm}$ for $30 \mathrm{sec}$.

3. Softbake for $3 \mathrm{~min}$ at $150^{\circ} \mathrm{C}$ (gives $15 \mu \mathrm{m}$ layer)

4. Repeat steps 2 and 3.

5. Expose

6. Post exposure bake at $175^{\circ} \mathrm{C}$ for $45 \mathrm{~min}$

7. Develop

8. Hardbake at $300^{\circ} \mathrm{C}$ for $30-60 \mathrm{~min}$.

Fig. 5 Modified polymer waveguide power divider and processing steps.
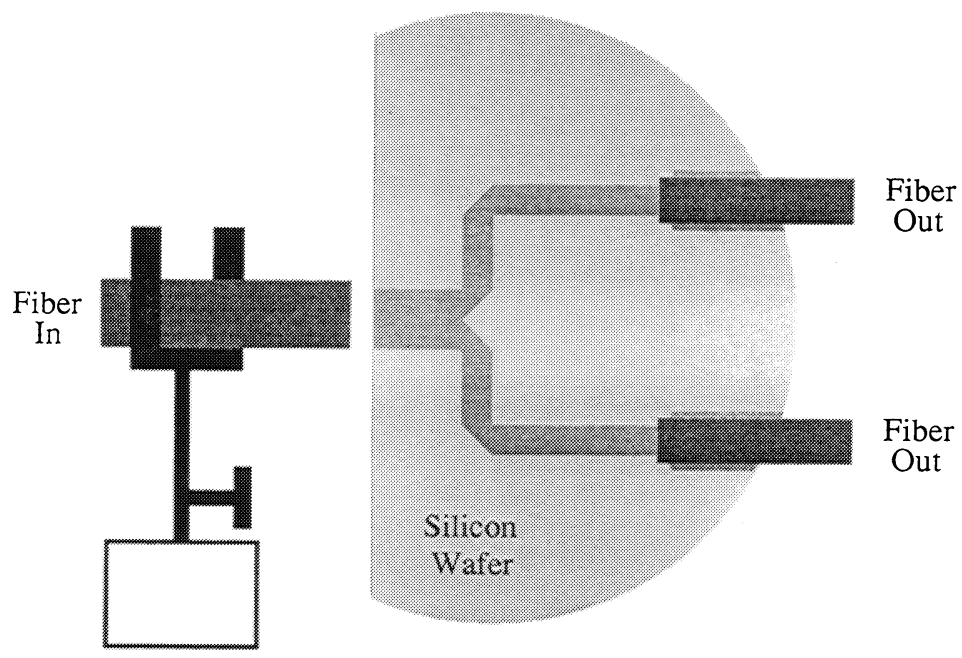

Micro-

Manipulator

Fig. 6 Fiber end fire coupling to the optical power divider. 